\title{
Negative Urinalyses in Febrile Infants Age 7 to 60 Days Treated for Urinary Tract Infection
}

\author{
Alan R Schroeder, MD'; Brian P Lucas, MD, MS²; Matthew D Garber, MD³; Russell J McCulloh, MD \\ Ashley A Joshi-Patel, DO5; Eric A Biondi, MD, MSBA ${ }^{6}$
}

\begin{abstract}
${ }^{1}$ Department of Pediatrics, Stanford University School of Medicine, Palo Alto, California; ${ }^{2}$ Department of Medicine, White River Junction Vermont VAMC and Geisel Medicine School at Dartmouth, Hanover, New Hampshire; ${ }^{3}$ Department of Pediatrics, University of Florida College of Medicine, Jacksonville, Florida; ${ }^{4}$ Department of Pediatrics, University of Kansas Medical Center, Kansas City, Missouri; ${ }^{5}$ Department of Pediatrics, Baylor College of Medicine, Houston, Texas; ${ }^{6}$ Department of Pediatrics, Johns Hopkins Medicine, Baltimore, Maryland.
\end{abstract}

The role of the urinalysis (UA) in the management of young, febrile infants is controversial. To assess how frequently infants are treated for urinary tract infection (UTI) despite having normal UA values and to compare the characteristics of infants treated for UTI who have positive versus negative UAs, we reviewed 20,570 wellappearing febrile infants 7-60 days of age evaluated at 124 hospitals in the United States who were included in a national quality improvement project. Of 19,922 infants without bacteremia and meningitis, 2,407 (12.1\%) were treated for UTI, of whom 2,298 (95.5\%) had an initial
UA performed. UAs were negative in 337/2,298 (14.7\%) treated subjects. The proportion of infants treated for UTI with negative UAs ranged from $0 \%-35 \%$ across hospitals. UA-negative subjects were more likely to have respiratory symptoms and less likely to have abnormal inflammatory markers than UA+ subjects, indicating that they are mounting less of an inflammatory response to their underlying illness and/or might have contaminated specimens or asymptomatic bacteriuria. Journal of Hospital Medicine 2019;14:101-104. (C) 2019 Society of Hospital Medicine

\section{T} he sensitivity of the urinalysis (UA) in young infants has been reported to be in the $75 \%$ to $85 \%$ range. ${ }^{1-4}$ This suboptimal sensitivity has prevented a widespread adoption of the UA as a true screening test for urinary tract infection (UTI). Although infants with a positive urine culture and a negative UA may have asymptomatic bacteriuria (AB) or contamination, ${ }^{5-7}$ they are often treated for UTI.

Due to these concerns, the American Academy of Pediatrics (AAP) recommended in their 2011 UTI Practice Guidelines that UA criteria should be incorporated into the definition of UTI. ${ }^{1}$ However, these guidelines were intended for the 2-24 month age range, leaving a gap in our understanding of the appropriate management of infants $<2$ months. It is unknown how UA results influence the current management of UTI in young, febrile infants. Using data from a large, nationally representative quality improvement project surrounding the management of febrile infants, this investigation aimed to examine how frequently infants are treated for UTI despite having normal UAs and to determine whether infant and hospital characteristics are different in infants treated for UTI with a positive UA as compared to those treated for UTI with a negative UA.

\footnotetext{
*Corresponding Author: Alan R. Schroeder, MD; E-mail aschroe@stanford.edu; Telephone: 650-725-0551
}

Received: August 16, 2018; Revised: October 4, 2018;

Accepted: October 31, 2018

C 2019 Society of Hospital Medicine DOI 10.12788/jhm.3120

\section{METHODS}

\section{Subjects and Setting}

This is a secondary analysis of the AAP's Reducing Excessive Variability in the Infant Sepsis Evaluation (REVISE) project that involved 20,570 well-appearing infants 7-60 days of age evaluated in the emergency department and/or inpatient setting for fever $\geq 38^{\circ} \mathrm{C}$ without a source between September 2015 and November 2017 at 124 community- and university-based hospitals in the United States. Data were collected via chart review and entered into a standardized tool for the project. This project was deemed exempt by the AAP Institutional Review Board. Because all data were de-identified, some sites did not require Institutional Review Board approval while others required data sharing agreements.

\section{Variables and Definitions}

A positive UA was defined as having any leukocyte esterase, positive nitrites, or $>5$ white blood cells (WBCs) per high power field. Treatment for UTI was defined using the question "Did the urine culture grow an organism that was treated as a pathogen with a full course of antibiotics?" Subjects treated for meningitis or bacteremia were excluded in order to focus on uncomplicated UTI. "Abnormal inflammatory markers" were defined as having a WBC count $<5,000$ or $>15,000$ cells $/ \mathrm{mm}^{3}$, an absolute band count $\geq 1,500$ cells $/ \mathrm{mm}^{3}$, a band to neutrophil ratio of $>0.2$, cerebrospinal fluid (CSF) WBC count of $>8 / \mathrm{mm}^{3}$, a positive CSF gram stain, or an elevated $\mathrm{C}$-reactive protein or procalcitonin level, as defined by the institutional range. Although technically not an "inflammatory 
TABLE. Characteristics of 2,298 Febrile Infants Treated for Urinary Tract Infection

\begin{tabular}{|c|c|c|c|c|}
\hline Characteristics & $\begin{array}{l}\text { Treated for UTI, UA-Negative } \\
\qquad(\mathrm{n}=337)\end{array}$ & $\begin{array}{l}\text { Treated for UTI, UA-Positive } \\
\qquad(\mathrm{n}=1,961)\end{array}$ & $\begin{array}{c}\text { aOR for Treatment } \\
\text { of UTI with Negative UA } \\
\text { (95\% Confidence Interval) }\end{array}$ & Adjusted $P$ Value \\
\hline \multicolumn{5}{|l|}{ Age } \\
\hline 7 to 30 days & $154(45.7 \%)$ & $810(41.3 \%)$ & $1.3(1.02-1.7)$ & .04 \\
\hline \multicolumn{5}{|l|}{ Sex } \\
\hline Boy & $222(65.9 \%)$ & $1,193(60.8 \%)$ & $1.2(0.9-1.6)$ & \\
\hline Girl & $115(34.1 \%)$ & $768(39.2 \%)$ & Referent $^{b}$ & .17 \\
\hline \multicolumn{5}{|c|}{ Abnormal inflammatory marker ${ }^{c}$} \\
\hline Yes & $123(38.4 \%)$ & $1,242(65.6 \%)$ & $0.3(0.3-0.4)$ & $<.001$ \\
\hline No & $244(72.4 \%)$ & $1,617(82.5 \%)$ & Referent $^{b}$ & \\
\hline \multicolumn{5}{|l|}{ Hospitals } \\
\hline \multicolumn{5}{|l|}{ University-affiliated } \\
\hline Yes & $219(65.0 \%)$ & $1,338(68.2 \%)$ & $0.9(0.6-1.3)$ & .63 \\
\hline No & $118(35.1 \%)$ & $623(31.8 \%)$ & Referent $^{b}$ & \\
\hline \multicolumn{5}{|l|}{ Urban setting } \\
\hline Yes & $240(71.2 \%)$ & $1,455(74.2 \%)$ & $0.8(0.6-1.2)$ & .32 \\
\hline No & $97(28.8 \%)$ & $506(25.8 \%)$ & Referent ${ }^{b}$ & \\
\hline South & $137(40.7 \%)$ & $725(37.0 \%)$ & Referent $^{b}$ & .002 \\
\hline Midwest & 77 (22.9\%) & $460(23.5 \%)$ & $0.9(0.6-1.4)$ & \\
\hline Northeast & $71(21.1 \%)$ & $280(14.3 \%)$ & $1.4(0.9-2.3)$ & \\
\hline West & $52(15.4 \%)$ & $496(25.3 \%)$ & $0.5(0.3-0.8)$ & \\
\hline
\end{tabular}

${ }^{a}$ Mixed-effects model was used to adjust for clustering by site. Due to missing inflammatory marker data for 84 (3.7\% of 2,298) children, the adjusted odds ratios were generated from 2,214 children.

bReferent odds for a 31 to 60-day old girl without elevated inflammatory markers or respiratory symptoms, who was cared for in an urban, university-affiliated hospital in the south with an annual volume of less than 50 febrile infants was 0.15 ( $95 \% \mathrm{Cl} 0.06$ to 0.35$)$.

cDefined as white blood cell count $<5,000$ or $>15,000$ cells $/ \mathrm{mm}^{3}$; cerebrospinal fluid white blood cell count $>8 / \mathrm{mm}^{2}$; positive cerebrospinal fluid gram stain; or elevated C-reactive protein or procalcitonin per institutional range. Inflammatory markers were missing for $17(5.0 \%)$ children with UA-negative UTI and $67(3.4 \%)$ children with UA-positive UTI (P-value $=0.14$ for difference in proportion).

Abbreviations: aOR, adjusted odds ratio; UA, urinalysis; UTI, urinary tract infection.

marker," CSF gram stain was included in this composite variable because in the rare cases that it is positive, the result would likely influence risk stratification and immediate management. Infants' ages were categorized as either 7-30 days or 31-60 days. Hospital length-of-stay (LOS) was recorded to the nearest hour and infants who were not hospitalized were assigned a LOS of 0 hours. Hospital characteristics were determined through a survey completed by site leads.

\section{Statistics}

Proportions were compared using chi-square test. We used multilevel mixed-effects logistic regression to determine associations between patients and hospital characteristics and UA-positivity in subjects treated for UTI. We accounted for the hospital clustering effect with a random effect that did not vary with patient characteristics. We "marginalized" the regression coefficients to reflect the average effect across hospitals. $8,9 \mathrm{We}$ 
$40 \%$

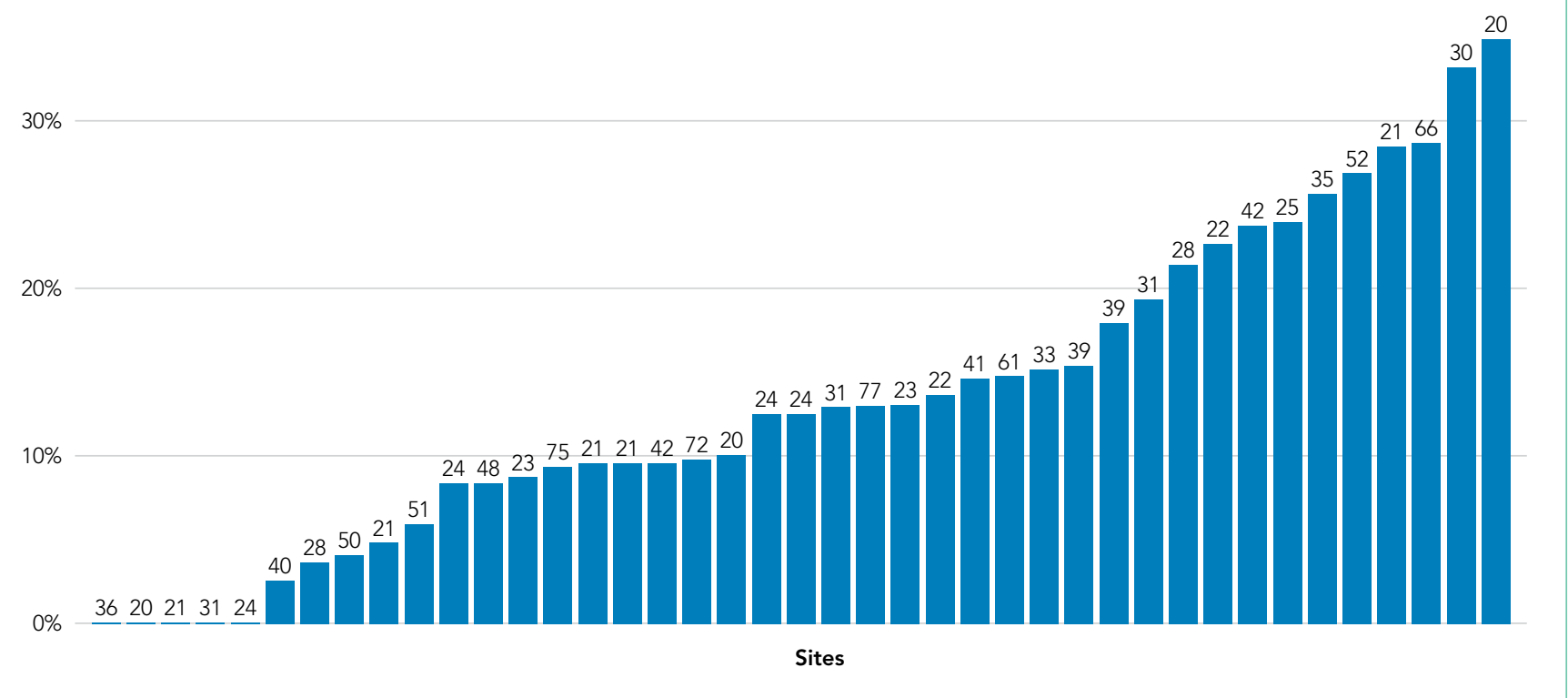

FIG. Proportion of Febrile Infants 7 to 60 Days Old Treated Having a UTI with Negative Urinalysis at 41 Sites with 20 or More UTI Cases

tested the overall importance of the hospital clustering effect on the treatment by comparing our multilevel model to a single-level model without hospital random effects using the likelihood ratio test.

\section{RESULTS}

A total of 20,570 infants from 124 hospitals were enrolled in the REVISE project, and 648 (3.2\%) were treated for bacteremia and/or meningitis. Of the remaining 19,922 infants, 2,407 (12.1\%) were treated for UTI, of whom 2,298 (95.5\%) had an initial UA performed. Urine cultures were obtained by catheterization or suprapubic aspirate in $90.3 \%$ and "other/unknown" in $9.7 \%$ of these 2,298 subjects.

UAs were negative in 337/2,298 (14.7\%) treated subjects. UA-negative subjects were more likely to be 7-30 days old (adjusted odds ratio [aOR] 1.3, 95\% $\mathrm{Cl} 1.02-1.7)$ and have upper respiratory symptoms (aOR 1.7, 95\% $\mathrm{Cl} 1.3-2.3$ ) and were less likely to have abnormal inflammatory markers (aOR 0.3, 95\% Cl 0.3-0.4) than UA+ subjects (Table). Even after accounting for the hospital characteristics depicted in the Table, treatment of UA-negative UTI was affected by the hospital $(P<.001)$, and the intraclass correlation coefficient was $6 \%(95 \% \mathrm{Cl}, 3 \%$ to $14 \%)$. The Figure illustrates substantial site variability in the proportion of infants treated for UTIs that were UA-negative, ranging from $0 \%$ to $35 \%$ in hospitals with $\geq 20$ UTI cases.

There was no significant difference in the proportion of catheterized specimens in infants treated for UTIs with negative versus positive UAs ( $90 \%$ vs $92 \%, P=.26$ ). The median hospital (interquartile range) LOS in infants treated for UTI with positive UAs was 58 (45-78) hours, compared to 54 (38-76) hours in infants treated for UTI with negative UAs and 34 (0-49) hours in infants who were not treated for UTI, meningitis, or bacteremia.

\section{DISCUSSION}

In this large, nationally representative sample of febrile infants 7-60 days of age, we demonstrate that nearly $15 \%$ of young febrile infants who are treated for UTIs have normal UAs. This proportion varied considerably among hospitals, suggesting that there are institutional differences in the approach to the UA. Infants treated for UA-negative UTIs were more likely to have respiratory symptoms and less likely to have abnormal inflammatory markers than infants treated for UA-positive UTIs, indicating that these infants are either developing a milder inflammatory response to their underlying illness and/or might not have true UTIs (eg due to AB or contamination).

The AAP recently updated their UTI practice parameter to recommend inclusion of UA results as diagnostic criteria for UTI. ${ }^{1}$ However, the fact that these guidelines do not include infants $<2$ months creates a gap in our understanding of the appropriate diagnostic criteria in this age group, as reflected by the site variability demonstrated in our investigation. The fact that up to $35 \%$ of infants treated for UTI at these different sites have normal UAs suggests that many practitioners continue to treat positive urine cultures regardless of UA values.

Several prior studies provide insight into the clinical significance of a positive urine culture in the absence of pyuria. Wettergren et al. $6,7,10$ reported growth from suprapubic aspirate in $1.4 \%$ of infants who were screened periodically with urine cultures obtained by bag at well-child checks over the course of the first year (with a point prevalence as high as $1.5 \%$ in boys aged 0.25 to 1.9 months)..$^{10}$ These infants were not more likely to have subsequent $\mathrm{UTI}^{7}$ or renal damage ${ }^{6}$ than infants without asymptomatic growth, leading the authors to conclude that this growth likely represented $A B$. These findings empha- 
size that the probability of a positive urine culture in any infant, even asymptomatic infants, is not insignificant.

Hoberman et al. ${ }^{11}$ demonstrated that dimercaptosuccinic acid scans did not reveal signs of pyelonephritis in 14/15 children $<2$ years of age with urine cultures growing $>50,000$ CFU/ $\mathrm{mL}$ but no pyuria on $U A$, and concluded that $A B$ was the most likely explanation for this combination of findings. Schroeder et al..$^{5}$ and Tzimenatos et al. ${ }^{12}$ examined infants $<2-3$ months with UTI and bacteremia caused by the same organism (and hence a true infection that cannot be explained by $A B$ or contamination) and demonstrated that the UA sensitivity in this population was $99.5 \%$ and $100 \%$, respectively, suggesting that the prior lower estimates of UA sensitivity in UTI in general, may have been biased by inclusion of positive urine cultures that did not represent UTI.

On the other hand, Shaikh et al. ${ }^{13}$ recently demonstrated that the sensitivity of the UA appears to vary by organism, with lower reported sensitivity in non-Escherichia coli organisms, leading the authors to conclude that this variability is evidence of suboptimal UA sensitivity. However, an alternative explanation for their findings is that non-E coli organisms may be more likely to cause $A B$ or contamination. ${ }^{14}$ The fact that follow-up suprapubic aspirates on infants with untreated catheterized cultures yielding these organisms are often negative supports this alternative explanation. ${ }^{15}$

The median LOS in infants with UA-negative UTI was nearly one day longer than infants not treated for serious bacterial infection. These infants may have also undergone urinary imaging and possibly prophylactic antibiotics, indicating high resource burden created by this subgroup of infants. Expanding AAP UTI guidelines to infants $<2$ months of age would likely reduce resource utilization, but continued research is needed to assess the safety of this approach. Young infants have immature immune systems and may not develop a timely inflammatory response to UTI, which raises concerns about missing bacterial infections.

Our investigation has several strengths, including the large, nationally representative sample that includes both children's and non-children's hospitals. Similar febrile infant investigations of this size have previously been possible only using administrative databases, but our investigation required chart review for all enrolled infants, ensuring that the subjects were febrile, well-appearing, and were treated for UTI. However, our findings are limited in that data were collected primarily as part of a quality improvement initiative, and some of our thresholds for "abnormal" laboratory values might be controversial. For example, urine WBC thresholds differ across studies, and our CSF WBC threshold of $>8 / \mathrm{mm}^{3}$ may be somewhat low given prior reports that values slightly above this threshold might be normal in infants under one month of age. ${ }^{16}$ The original intent of the inflammatory marker composite variable was to aid in risk stratification, but we were unable to collect granular data for all potentially relevant variables. In planning the REVISE project, we attempted to create straightforward, unambiguous variables to facilitate the anticipated high volume of chart reviews. Although patients categorized as having UTI might not have had true UTIs, by linking the "UTI" variable to practitioner management (rather than UA and microbiologic definitions), our data reflect real-world practice.

\section{Acknowledgments}

The authors would like to thank all of the site directors who participated in the REVISE project, and Brittany Jennings, Naji Hattar, Faiza Wasif, and Vanessa Shorte at the American Academy of Pediatrics for their leadership and management.

Disclosures: Dr. Schroeder has received honoraria for grand rounds presentations on the subject of urinary tract infections, and Dr. Biondi has received consulting fees from McKesson Inc. The other authors have no financial relationships to disclose.

\section{References}

1. Roberts KB. Urinary tract infection: Clinical practice guideline for the diagnosis and management of the initial UTI in febrile infants and children 2 to 24 months. Pediatrics. 2011;128(3):595-610. doi: 10.1542/peds.2011-1330.

2. Bachur R, Harper MB. Reliability of the urinalysis for predicting urinary tract infections in young febrile children. Arch Pediatr Adolesc Med. 2001;155(1):60. doi: 10.1001/archpedi.155.1.60.

3. Bonadio W, Maida G. Urinary tract infection in outpatient febrile infants younger than 30 days of age. Pediatr Infect Dis J. 2014;33(4):342-344. doi: 10.1097/inf.0000000000000110.

4. Hoberman A, Wald ER. Urinary tract infections in young febrile children. Pediatr Infect Dis J. 1997;16(1):11-17. doi: 10.1097/00006454-199701000-00004.

5. Schroeder AR, Chang PW, Shen MW, Biondi EA, Greenhow TL. Diagnostic accuracy of the urinalysis for urinary tract infection in infants $<3$ months of age. Pediatrics. 2015;135(6). doi: 10.1542/peds.2015-0012d.

6. Wettergren B, Hellstrom M, Stokland E, Jodal U. Six-year follow up of infants with bacteriuria on screening. BMJ. 1990;301(6756):845-848. doi: 10.1136/ bmj.301.6756.845.

7. Wettergren B, Jodal U. Spontaneous clearance of asymptomatic bacteriuria in infants. Acta Paediatrica. 1990;79(3):300-304. doi: 10.1111/j.16512227.1990.tb11460.x

8. Hedeker D, Toit SHCD, Demirtas H, Gibbons RD. A note on the marginalization of regression parameters from mixed models of binary outcomes. Biometrics. 2017;74(1):354-361. doi: 10.1111/biom.12707.

9. Neuhaus JM, Kalbfleisch JD, Hauck WW. A comparison of cluster-specific and population-averaged approaches for analyzing correlated binary data. Int Stat Rev. 1991;59(1):25. doi: 10.2307/1403572.

10. Wettergren $B$, Jodal $U$, Jonasson $G$. Epidemiology of bacteriuria during the first year of life. Acta Paediatrica. 1985;74(6):925-933. doi: 10.1111/j.16512227.1985.tb10059.x

11. Hoberman A, Wald ER, Reynolds EA, Penchansky L, Charron M. Is urine culture necessary to rule out urinary tract infection in young febrile children? Pediatr Infect Dis J. 1996;15(4):304-309. doi: 10.1097/00006454-19960400000005.

12. Tzimenatos $L$, Mahajan $P$, Dayan PS, et al. Accuracy of the urinalysis for urinary tract infections in febrile infants 60 days and younger. Pediatrics. 2018;141(2). doi: 10.1542/peds.2017-3068.

13. Shaikh N, Shope TR, Hoberman A, Vigliotti A, Kurs-Lasky M, Martin JM. Association between uropathogen and pyuria. Pediatrics. 2016;138(1). doi: 10.1542/peds.2016-0087

14. Schroeder AR. UTI and faulty gold standards. Pediatrics. 2017;139(3). doi: 10.1542/peds.2016-3814a.

15. Eliacik K, Kanik A, Yavascan $O$, et al. A comparison of bladder catheterization and suprapubic aspiration methods for urine sample collection from infants with a suspected urinary tract infection. Clinical Pediatrics. 2016;55(9):819824. doi: 10.1177/0009922815608278.

16. Thomson J, Sucharew H, Cruz AT, et al. Cerebrospinal fluid reference values for young infants undergoing lumbar puncture. Pediatrics. 2018;141(3). doi: 10.1542/peds.2017-3405. 